\title{
Condiciones de trabajo y satisfacción laboral de las enfermeras del Complejo Hospitalario PNP Luis N Sáenz
}

Working conditions and job satisfaction of the nurses of the Hospital Complex PNP Luis N Sáenz

Condições de trabalho e satisfação profissional dos enfermeiros do Complexo Hospitalar PNP Luis N Sáenz

\section{ARTÍCULO GENERAL}

\section{Milagros Lissette Millones Trinidad \\ mili_libra16@hotmail.com}

\section{Servicio de Pediatría del Complejo Hospitalario PNP Luis N Sáenz}

Recibido 25 de Diciembre 2021 | Arbitrado y aceptado 25 de Diciembre 2021 | Publicado el 28 de Enero 2022

\section{RESUMEN}

Las condiciones de trabajo son condicionantes a la presencia de una satisfacción e insatisfacción laboral, que van a repercutir en su calidad de vida; por ende, en la actualidad es fundamental que los profesionales de enfermería laboren en ambientes de satisfacción a fin de que sean unos de los indicadores para que su desempeño profesional sea de calidad asistencial. El objetivo del estudio es determinar la relación que existe entre las condiciones de trabajo y la satisfacción laboral de los enfermeros del Complejo Hospitalario PNP Luis N Sáenz. El diseño aplicado fue cuantitativo, descriptivo, corte transversal y correlacional. Se encuestaron a 331 enfermeros, donde el $41.1 \%$ tienen más de 50 años, $36.6 \%$ entre 25 a 39 años y $22.4 \%$ entre 40 a 49 años; $87.9 \%$ son mujeres y $12.1 \%$ son varones. En dos modelos de regresión se pudo observar que las condiciones de trabajo físicas $y$ psicológicas se encuentran negativamente asociadas con la satisfacción intrínseca; mientras que las condiciones sociales se encuentran positivamente asociadas con la satisfacción extrínseca. Se discuten las diferencias en las condiciones de trabajo y sus asociaciones con la satisfacción laboral. Palabras Clave: Condiciones de trabajo, satisfacción laboral, enfermeros

\section{ABSTRACT}

Working conditions are determining factors for the presence of job satisfaction and dissatisfaction, which will affect their quality of life; therefore, it is currently essential that nursing professionals work in environments of satisfaction so that they are one of the indicators for their professional performance to be of quality care. The objective of the study is to determine the relationship between working conditions and job satisfaction of nurses at the PNP Luis N Sáenz Hospital Complex. The applied design was quantitative, descriptive, cross-sectional and correlational. 331 nurses were surveyed, where $41.1 \%$ are over 50 years old, $36.6 \%$ between 25 and 39 years old and $22.4 \%$ between 40 and 49 years old; $87.9 \%$ are women and $12.1 \%$ are men. In two regression models it was observed that physical and psychological working conditions are negatively associated with intrinsic satisfaction; while social conditions are positively associated with extrinsic satisfaction. Differences in working conditions and their associations with job satisfaction are discussed.

Keywords: Working conditions, job satisfaction, nurses.

\section{RESUMO}

As condições de trabalho são fatores determinantes para a presença de satisfação e insatisfação no trabalho, o que afetará sua qualidade de vida; portanto, atualmente é fundamental que os profissionais de enfermagem trabalhem em ambientes de satisfação para que sejam um dos indicadores para que sua atuação profissional seja de qualidade assistencial. $\mathrm{O}$ objetivo do estudo é determinar a relação entre as condições de trabalho e a satisfação profissional dos enfermeiros do Complexo Hospitalar PNP Luis N Sáenz. O delineamento aplicado foi quantitativo, descritivo, transversal e correlacional. Foram pesquisados 331 enfermeiros, onde $41,1 \%$ têm mais de 50 anos, $36,6 \%$ entre 25 e 39 anos e $22,4 \%$ entre 40 e 49 anos; $87,9 \%$ são mulheres e 12,1\% são homens. Em dois modelos de regressão observou-se que as condições físicas e psicológicas de trabalho estão negativamente associadas à satisfação intrínseca; enquanto as condições sociais estão positivamente associadas à satisfação extrínseca. Diferenças nas condições de trabalho e suas associações com a satisfação no trabalho são discutidas.

Palavras-chave: Condições de trabalho, satisfação no trabalho, enfermeiros. 


\section{Introducción}

Milagros Lissette Millones Trinidad

Los enfermeros son fundamentales para todos los entornos sanitarios por la atención sanitaria que brindan todos los días, pero también enfrentan muchos desafíos en el complejo entorno de atención médica actual; sin embargo, existen cinco grandes problemas o condiciones de trabajo que enfrentan los enfermeros como son la dotación de personal, las horas laborales largas, los riesgos laborales, los conflictos laborales, la intimidación y acoso en el lugar de trabajo.

La escasez de personal en los entornos hospitalarios se presenta en un $72 \%$ siendo una de las principales preocupaciones de los enfermeros, están preocupadas porque significa que no tienen el tiempo para brindar la atención que creen que es necesaria para los pacientes y sus familiares; el 34\% de los enfermeros reconocieron tiene un impacto negativo considerable o grande en la atención al paciente, dando como resultado errores, tasas más altas de morbilidad y mortalidad. Para ayudar a compensar la escasez de personal, los enfermeros a menudo deben trabajar en turnos prolongados en un 52\% con riesgo de fatiga que podría provocar errores, ya que no deberían trabajar más de 40 horas a la semana y más de 12 horas al día o menos si es posible, porque se llega al punto que se empieza a tener un deterioro cognitivo, por quedarse hasta tarde, trabajar horas extra o volver a trabajar después de dormir poco si su lugar de trabajo los necesita.

Asimismo, los enfermeros se enfrentan a una serie de peligros en el lugar de trabajo en un $89 \%$, como la exposición a patógenos transmitidos por la sangre, lesiones (objetos punzocortantes, aguja o bisturí), dermatitis relacionada con el lavado de manos y gérmenes del resfriado y gripe. Los conflictos en el lugar de trabajo en un 38\% también es un problema urgente para los enfermeros como son golpes, patadas y palizas fueron los incidentes más comunes denunciados; sin embargo, no siempre informan tales incidentes y una investigación limitada sobre el tema.

Los enfermeros también sufren intimidación y acoso en un $71 \%$ por un paciente y familiares, el $62 \%$ han tenido una experiencia personal con abuso físico o verbal en el trabajo, y una de cada cuatro han sido agredidos físicamente en el trabajo, también se producen entre compañeros y $45 \%$ de enfermeros han sido acosadas o intimidadas verbalmente por otros enfermeros.

La satisfacción laboral es un componente importante de la vida de los enfermeros que puede afectar la seguridad del paciente, moral del personal, productividad y desempeño, calidad de atención, retención y rotación, y compromiso con la organización; 
con tasas de rotación del 35\% al 55\% en el primer año de empleo, empleo en enfermería se redujo al $82 \%$ de 3 años después de carrera temprana; por ello, se ha demostrado que cuando la satisfacción laboral aumenta, la rotación disminuye, es dinámica y puede variar según características individuales, expectativas, estilo de gestión, cambios en políticas y elecciones de estilo de vida individuales; satisfacer las necesidades de los enfermeros es particularmente importante durante el inicio de la carrera, que tiene un impacto dramático en la contribución de los enfermeros a largo plazo.

En las instituciones con poco personal, es bastante común que se les pida a los enfermeros que permanezcan en un turno doble, puede ir en detrimento de la calidad de atención al paciente, por trabajar en turnos de más de 13 horas aumenta la insatisfacción de pacientes con la atención; además, los enfermeros que trabajaban más de 10 horas tenían 3 veces más probabilidades de informar insatisfacción laboral y síntomas de agotamiento, lo que genera un gran estrés, muchas situaciones, esto conduce a una alta rotación de puestos de trabajo para la institución de salud.

El número de pacientes por enfermeros tiene un efecto enorme no solo del entorno laboral, sino también en el desempeño de los pacientes, incluso puede ser una cuestión de vida o muerte; por ende, la insatisfacción laboral equivale a una baja atención al paciente, puede parecer obvio que los profesionales de enfermería que no disfrutan de su trabajo no se desempeñan mejor; que un aumento del $25 \%$ en el disfrute del trabajo de enfermería durante un período de dos años estaba relacionado con un aumento general de la calidad de la atención entre el 5 y el $20 \%$.

Por todo ello, un mejor ambiente de trabajo tiene menos errores, porque los enfermeros que no estaban satisfechos con sus condiciones de trabajo y sentían que no había oportunidades de avance profesional disponibles, se encomendó la tarea de implementar una serie de estrategias para ayudar a cambiar las cosas, sino también para los pacientes; asimismo, no hubo un aumento en la comunicación con el paciente y disminuye una serie de indicadores de calidad de atención. la productividad disminuye, y produciendo errores en la administración de medicamentos.

Los enfermeros sobre la satisfacción laboral y percepciones de las condiciones laborales ofrecen un barómetro organizacional de cómo les está yendo a los pacientes; asimismo, la satisfacción del paciente es mucho menor en las instituciones donde muchas enfermeras se sienten agotadas e insatisfechas con sus condiciones de trabajo que en otras instituciones, puede ser posible mejorar la satisfacción del paciente y evitar resultados 
adversos para el paciente, al mismo tiempo mejora la satisfacción y retención de enfermeros al mejorar las condiciones de trabajo.

La satisfacción laboral es un factor importante en el compromiso de los enfermeros que probablemente hagan los miembros del equipo de salud; sin duda, el desempeño laboral se ve afectado cuando no se sienten satisfechas con su trabajo, por no hablar de su calidad de vida; que depende de varios factores como satisfacción con el salario, oportunidades de promoción, beneficios complementarios, seguridad laboral, relación con los compañeros y los supervisores, lo que conduce a la reducción de ausencias, errores de tareas, conflictos en el trabajo y rotación.

Dado que el trabajo es un aspecto importante de la vida de las personas y la mayoría de las personas pasan gran parte de su vida laboral en el trabajo, comprender los factores que intervienen en la satisfacción laboral es fundamental para mejorar el rendimiento y la productividad de los empleados, en especial de los profesionales de enfermería asistenciales.

En el Complejo Hospitalario Luis N Sáenz PNP; se pudo evidenciar que los enfermeros laboran en ambientes inadecuados para desempeñar su función, infraestructura antigua, escaso personal asistencial, equipos obsoletos y materiales incompletos; por lo que, al interactuar manifestaron: "trabajo demasiado", "turnos inadecuados", "carencia de incentivos", "no percibo capacitaciones continuas", "no hay reconocimiento por mi labor", "no se dan las condiciones adecuadas en el trabajo", entre otras expresiones. Trayendo consigo ausentismo laboral, sobrecarga laboral, estrés, desmotivación; en consecuencia, se requiere conocer qué está pasando con las enfermeras de esta institución, se hace necesario identificar las condiciones de trabajo que generan satisfacción e insatisfacción en su trabajo; por ende, en sus funciones asistenciales para brindar una atención de calidad.

El presente estudio es importante ya que las condiciones laborales optimas, libre de riesgos, clima laboral adecuado, excelentes relaciones interpersonales con su jefe y sus colegas, y salarios acordes a sus necesidades y expectativas; harán posible que perciban su labor asistencial diaria de forma satisfactoria, repercutiendo en su bienestar físico y mental, influirá en la atención de calidad, conllevando a un clima laboral propicio para motivarla en su productividad, de forma eficiente y eficaz, haciendo su calidad de vida laboral sea satisfactoria. 
Sin embargo, las condiciones laborales inaceptables, como bajos salarios, inestabilidad laboral, sobrecarga laboral, estrés, turnos nocturnos, trabajo rutinario, hacer guardias, trabajar en varios lugares e incluso descuidar su vida familiar; traerá consigo que se perciban una insatisfacción laboral, el cual repercutirá negativamente en su calidad asistencial, con aparición de fatiga, estrés laboral, enfermedades físicas, emocionales, conllevándolo a presentar a un bajo rendimiento laboral, escasa productividad y el ausentismo laboral.

Asimismo, el objetivo del presente estudio fue determinar la relación que existe entre las condiciones de trabajo y la satisfacción laboral de los enfermeros del Complejo Hospitalario PNP Luis N Sáenz, 2019.

\section{Metodología}

\section{Participantes}

El diseño del estudio fue cuantitativo, transversal y correlacional. La muestra fue obtenida por conveniencia apuntando a encuestar a todos los enfermeros del Complejo Hospitalario PNP Luis N Sáenz. En total se alcanzó a encuestar a 331 enfermeros con grado civil o policial, nombrado o contratado, con antigüedad de al menos año de servicio. Los enfermeros firmaron un consentimiento informado previamente a su participación, y fueron excluidos los enfermeros con cargo administrativo. En la tabla 1 se pueden observar las características descriptivas de la muestra, se observa que la mayoría de participantes del estudio fueron mayores de 40 años (63.44\%), también la mayoría fueron del sexo femenino (87.92\%). Con respecto al tiempo que laboran en el servicio la mayoría de ellos tiene entre 1 y 5 años (77.04\%), mientras que la mayoría de los participantes trabaja más de 5 años en la Policía Nacional del Perú (72.20\%).

\section{Tabla 1}

Frecuencias y porcentajes de las características de los participantes

\begin{tabular}{lcc}
\hline & $\mathrm{f}$ & $\%$ \\
\hline Edad & 121 & 36.56 \\
25 a 39 años & 210 & 63.44 \\
40 a más & & \\
Sexo & 40 & 12.08 \\
Masculino & 291 & 87.92 \\
Femenino & \\
Tiempo que labora en el servicio & 77.04 \\
Hasta 5 años & 255 & 22.96 \\
Más de 5 años & 76 &
\end{tabular}


Tiempo que labora en la institución

Hasta 5 años 92

Más de 5 años

\section{Instrumentos}

Condiciones de Trabajo: Se utilizó la Escala Condiciones de Trabajo (Blanch, Sahagun y Cervantes, 2010) adaptada por la Organización Internacional del Trabajo (OIT) y el Consejo Internacional de Enfermería (CIE); esta escala consta de 15 ítems de tipo Likert, y se divide en tres dimensiones: condiciones físicas, psicológicas y sociales. El nivel de confiabilidad reportado para esta escala es de un Alfa de Cronbach de 0.71.

Satisfacción laboral: Para esta escala se utilizó la Escala de Satisfacción Laboral General (Warr, Cook y Wall, 1979) adaptada por Pérez Bilbao (2006). Esta escala consta de 15 ítems en escala Likert. Esta escala se divide en dos dimensiones: satisfacción intrínseca y extrínseca. La confiabilidad de este instrumento se ha reportado con un Alfa de Cronbach de 0.89 .

\section{Procedimiento}

Para la recolección de datos se solicitó la autorización, mediante una carta de presentación dirigida al director del Complejo Hospitalario PNP Luis N. Sáenz; posteriormente a ello, se inscribió el estudio en la Oficina de Docencia e Investigación de la institución de salud. Luego se hizo la coordinación con los directivos del hospital a fin de coordinar con las participantes del estudio para realizar el cronograma de recolección de datos. Finalmente, se realizó la encuesta en el mes de marzo del 2021, en un lapso de 20 a 30 minutos por cada uno de los enfermeros.

\section{Plan de Análisis}

Inicialmente se realizaron análisis descriptivos y correlacionales de las variables de estudio. En esta etapa se revisó el supuesto de normalidad con la prueba de Shapiro-Willk donde ninguna de las variables mostró una distribución normal por lo que se utilizó el coeficiente Rho de Spearman para estimar las correlaciones. Posteriormente, para determinar la asociación entre las condiciones de trabajo y la satisfacción laboral controlando los efectos de las variables sociodemográficas, se estimaron dos modelos de regresión múltiple donde en un primer modelo se usó como variable de respuesta a la Satisfacción Intrínseca, como variables de control a la edad, el sexo y el tiempo en el servicio y como variables predictoras a las tres dimensiones de las condiciones laborales. 
Mientras que en un segundo modelo se usó como variable de respuesta a la Satisfacción Extrínseca, con las mismas variables de control y variables predictoras del primer modelo. Todos los análisis fueron llevados a cabo en el software R 4.1.0 (R Core Team, 2021).

\section{Resultados}

En la Tabla 2 se pueden observar los estadísticos descriptivos de las variables de estudio, se observa que, en cuanto a las condiciones laborales, las condiciones físicas son las que tienen el promedio más alto $(M=2.01, D E=0.28)$, mientras que el promedio más bajo es el de las condiciones sociales $(M=1.91, D E=0.43)$. Por otro lado, con respecto a la satisfacción laboral, el promedio de la satisfacción intrínseca es ligeramente más alto $(M=1.64, D E=0.21)$ que la satisfacción extrínseca $(M=1.88, D E=0.27)$. Con respecto las correlaciones de interés, se puede observar que la satisfacción intrínseca se correlaciona negativa y ligeramente a las condiciones físicas del trabajo $(R h o=-0.22, p$ $<0.001)$ y a las condiciones sociales $(R h o=-0.18, p<0.001)$, y se correlaciona negativa y fuertemente a las condiciones psicológicas $(R h o=-0.43, p<0.001)$. Por otro lado, la satisfacción extrínseca solo se correlaciona negativa y levemente con las condiciones psicológicas del trabajo $($ Rho $=-0.11, p<0.05)$.

Tabla 2.

Medias, Desviaciones Estándar y Correlaciones de Spearman entre las subescalas de condición laboral y satisfacción laboral

\begin{tabular}{|c|c|c|c|c|c|c|}
\hline & $\mathrm{M}$ & $\mathrm{DE}$ & 1 & 2 & 3 & 4 \\
\hline 1. C. T. Físicas & 2.01 & 0.28 & - & & & \\
\hline 2. C. T. Psicológicas & 1.59 & 0.18 & $0.14 * *$ & - & & \\
\hline 3. C. T. Sociales & 1.91 & 0.43 & $0.33 * * *$ & $0.28 * * *$ & - & \\
\hline 4. Satisfacción Intrínseca & 1.64 & 0.21 & $-0.22 * * *$ & $-0.43 * * *$ & $-0.18 * *$ & - \\
\hline 5. Satisfacción Extrínseca & 1.88 & 0.27 & 0.07 & $-0.11 *$ & 0.09 & $0.42 * * *$ \\
\hline
\end{tabular}

En la Tabla 3 se pueden observar los modelos de regresión estimados para determinar la asociación entre las condiciones de trabajo y la satisfacción laboral. En el modelo de la satisfacción intrínseca se puede observar que ninguna de las variables de control se encuentra asociadas a la satisfacción intrínseca, mientras que para las condiciones de trabajo se observa que las condiciones físicas se predicen negativa y levemente $(b=-0.10, p=0.03, B=-0.13)$ la satisfacción intrínseca; por otro lado, las condiciones psicológicas tienen un efecto predictivo negativo y más grande sobre la 
satisfacción intrínseca $(b=-0.50, p<0.001, B=-0.42)$. En el modelo de la satisfacción extrínseca se puede observar que los participantes de mayor edad tienen menos satisfacción extrínseca que los participantes más jóvenes $(b=-0.13, p<0.001, B=-0.23)$, sin embargo, los participantes que llevan más tiempo en el servicio tienen más satisfacción extrínseca que los que llevan menos de 6 años en el servicio $(b=0.27, p<$ $0.001, B=0.43$ ); finalmente, las condiciones de trabajo sociales predicen leve y positivamente la satisfacción extrínseca $(b=0.09, p=0.02, B=0.14)$.

Tabla 3.

Modelos de regresión múltiple para la predicción de satisfacción laboral

\begin{tabular}{lllllll}
\hline & \multicolumn{3}{l}{ Satisfacción Intrínseca } & \multicolumn{3}{l}{ Satisfacción Extrínseca } \\
\cline { 2 - 7 } & $\mathrm{b}$ & $\mathrm{EE}$ & $\mathrm{B}$ & $\mathrm{b}$ & $\mathrm{EE}$ & $\mathrm{B}$ \\
\hline Intercepto & $18.53^{* * *}$ & 0.12 & & $2.21 * * *$ & 0.15 & \\
Edad (40 años a más) & 0.03 & 0.03 & 0.07 & $-0.13 * * *$ & 0.04 & -0.23 \\
Sexo (Femenino) & -0.03 & 0.03 & -0.05 & -0.07 & 0.04 & -0.09 \\
T. S. (más de 6 años) & 0.00 & 0.03 & -0.01 & $0.27 * * *$ & 0.04 & 0.43 \\
C. T. Físicas & $-0.10^{*}$ & 0.05 & -0.13 & -0.06 & 0.06 & -0.07 \\
C. T. Psicológicas & $-0.50^{* * *}$ & 0.08 & -0.42 & -0.18 & 0.09 & -0.12 \\
C. T. Sociales & 0.00 & 0.03 & 0.00 & $0.09 *$ & 0.04 & 0.14 \\
\hline$* \mathrm{p}<0.05, * * \mathrm{p}<0.01, * * * \mathrm{p}<0.001$ & & & & &
\end{tabular}

\section{Discusión y Conclusiones}

Los resultados evidencian que las condiciones de trabajo físicas y sociales del complejo hospitalario no se encuentran directamente relacionadas con la satisfacción laboral intrínseca; es decir, no existe una relación significativa entre lo que respecta trabajar en un ambiente laboral libre de riesgos físicos, el contar con equipos tecnológicos seguros, entre otros, y el hecho de tener más o menos condicionantes motivacionales o satisfactorios y sentimientos relacionados con el crecimiento o desarrollo personal, que finalmente generarían una complacencia en el ámbito laboral que haría que la labor asistencial de los enfermeros sea de gran productividad. Estos resultados difieren de la investigación de Ore y Soto (2019), ya que al estudiar el nivel de satisfacción de enfermeros que trabajaban en un hospital en relación con las condiciones laborales, estos mostraban significativa relación al evidenciar un incremento de la satisfacción laboral a medida que las condiciones laborales mejoraban.

Con respecto a las condiciones de trabajo psicológicas y el nivel de satisfacción laboral intrínseco, podemos observar que ambas variables están relacionadas 
Milagros Lissette Millones Trinidad

significativamente, con esto, en cuanto las condiciones psicológicas son mayores; es decir, cuando existen más casos de condiciones psicológicas inadecuadas en el trabajo, el nivel de satisfacción laboral intrínseco, disminuye. Los hallazgos encontrados son los mismos que evidenció Tapia, S.V. (2017) en su trabajo de investigación, quien estudió los factores de riesgo psicosociales y la satisfacción laboral en enfermeras del Instituto Nacional de Salud del Niño en Lima, para lo cual determinó que cuando existía mayor presencia de factores de riesgo psicosociales en el trabajo, la satisfacción laboral de las enfermeras era igualmente menor. Estos descubrimientos siguen la línea de lo explicado por la Organización Internacional del Trabajo (2018), la cual refiere que las adecuadas condiciones psicológicas de trabajo en los profesionales de enfermería, son esenciales para que estos trabajadores puedan desempeñarse correctamente en su campo laboral, lo que involucra principalmente, por ejemplo, el asistir de manera tranquila y satisfactoria a los pacientes. Al tener menos carga mental, a pesar de las exigencias diarias y las jornadas laborales extenuantes, los trabajadores verán motivada su productividad, haciendo que su calidad de vida laboral se lleve a cabo con menor esfuerzo.

Por otro lado, se puede observar que a medida que las condiciones sociales de trabajo sean mejores para los enfermeros del complejo hospitalario, el nivel de satisfacción laboral extrínseco de los mismos, también incrementa. Estos resultados concuerdan con los de Campos, G. et al (2018), el cual determinó en su estudio que un grupo de enfermeras que trabajaban en un instituto especializado en Perú, manifestaban una relación positiva entre las dimensiones sociales que se presentaban en su centro de trabajo con respecto al nivel de satisfacción laboral, de tal manera que a medida que las dimensiones sociales aumentaban, también lo hacía la satisfacción laboral. Estos hallazgos parecen tener sentido con lo que manifiesta La Organización Internacional del Trabajo (2018), quien explica que las condiciones de trabajo sociales son determinantes para que el profesional de enfermería se sienta cómodo y satisfecho con su trabajo.

Ahora, es conveniente mencionar que las estadísticas nos refieren, además, que las condiciones de trabajo tanto físicas como sociales, no se encuentran relacionadas con el nivel de satisfacción laboral. Luengo, C. y Sanhueza, O. (2016) exponen resultados que difieren con los del presente estudio, ya que demuestran que cuando las condiciones laborales físicas; sociales, entre otras, son deficientes, el personal de enfermería de un centro médico, expresa insatisfacción laboral, lo que se manifiesta mediante la afectación de la salud tanto física como mental de los profesionales. Asimismo, existe una menor satisfacción extrínseca por parte del personal de mayor edad en comparación con los más 
Milagros Lissette Millones Trinidad

jóvenes; sin embargo, los que tienen más tiempo en el servicio, presentan mayor satisfacción que los que llevan menos de seis años. Resultados similares son los que reportan Ríos, M.L. y Godoy, C. (2008) al poner de manifiesto que, a medida que la edad se iba incrementando en un grupo de profesionales de enfermería, el nivel de satisfacción laboral general también iba descendiendo; sin embargo, difieren con nuestro estudio al encontrar que mientras más antiguo era el personal, estos eran más exigentes en cuanto a la percepción que tenían sobre la satisfacción en su centro laboral. Esto, se explicaba, era probablemente debido a que a medida que un profesional iba creciendo tanto en edad como en experiencia y conocimientos, se volvía más riguroso y crítico con respecto a la valoración de su trabajo, entorno y demás.

A modo de conclusión, se puede decir que debido a que los resultados demuestran que, no en todos los casos las condiciones de trabajo están estrechamente relacionadas con el nivel de satisfacción laboral, se recomienda realizar mayores trabajos de investigación que puedan explicar o determinar las posibles causas de estos hallazgos y a qué estarían relacionadas. De la misma manera, tomar en cuenta los resultados presentados por esta investigación para brindar mayor importancia a la estrecha relación que existe entre las condiciones de trabajo psicológicas y el descenso del nivel de satisfacción laboral del personal de enfermería, con el fin de evaluar y analizar los motivos que la causan y tomar iniciativas para mejorarla, lo que en consecuencia podría resultar con el hecho de contar con un equipo de trabajadores más satisfechos con su labores y por ende, ejercer un mejor desempeño laboral.

\section{Referencias Bibliográficas}

Aguirre D (2016). Factores que afectan la satisfacción laboral de los recursos humanos de enfermería. Habana-Cuba: Instituto Superior de Ciencias Médicas de La Habana.

Atalaya M (2017). Satisfacción laboral y productividad en los hospitales. Barcelona: Revista de psicología de la Escuela de Organización Industrial/EOI.

Canales M, Valenzuela S, Paravic T (2016). Condiciones de trabajo de los profesionales de enfermería en Chile (Tesis). Santiago-Chile: Universidad de Concepción.

Ceballos S, Valenzuela T, Paravic T (2016). Factores de riesgos psicosociales en el trabajo de enfermería (Tesis). Santiago-Chile: Universidad de Concepción de Chile.

Churo M (2016). Satisfacción laboral en el personal administrativo del Área de los Recursos Humanos, de la Dirección del Hospital Honorio Delgado que es parte del 
sector del Ministerio de Salud. Arequipa-Perú: MINSA.

Herzberg F (2016). Factores de la satisfacción laboral: Herzberg y su teoría de la motivación e higiene. Medellín-Colombia: Revista Universidad EAFIT.

Herzberg F (2016). Teoría motivacional de Herzberg o teoría de los factores motivacionales o satisfactorios. Medellín-Colombia: Revista Universidad EAFIT.

Herzberg F (2016). Teoría motivacional de Herzberg ó teoría de los factores higiénicos o insatisfactorios. Medellín-Colombia: Revista Universidad EAFIT.

Instituto Nacional de Seguridad e Higiene en el Trabajo (2018). Condiciones de trabajo en el ámbito sanitario. Madrid-España: INSHT.

Instituto Nacional de Seguridad y Salud en el Trabajo. Riesgos laborales a nivel mundo por las condiciones de trabajo de enfermería. Madrid-España: INSHT.

Irazábal U, Martín R (2017). Condiciones de las condiciones de trabajo en la organización. Madrid-España: Diccionario empresarial Wolterskluwer.

Llop A, Tarafa G, Benach J (2018). Personal de enfermería, condiciones de trabajo y su impacto en la salud (Tesis). Madrid-España: Universidad Autónoma de Madrid.

Locke E (2018). La naturaleza y causas de la satisfacción en el trabajo: manual de psicología industrial y organizacional. Chicago-Estados Unidos: Rand McNally College.

Loli R (2016). Nivel de satisfacción laboral del profesional de enfermería en el servicio de centro quirúrgico Hospital Nacional Alberto Sabogal Sologuren. EsSalud. Tesis de Especialista en Enfermería en Centro Quirúrgico. Lima-Perú: Universidad Nacional Mayor de San Marcos.

Luengo C, Sanhueza O (2018). Condiciones de trabajo y calidad del cuidado del profesional de enfermería (Chile). Concepción-Chile: Universidad de Concepción.

Marriner A, Raile M (2017). Modelos y teorías de enfermería: teoría del entorno de Florence Nightingale. Barcelona-España: Elsevier.

Martínez L, Oviedo O, Luna C (2016). Condiciones de trabajo que impactan en la vida laboral (Tesis). Barranquilla-Colombia: Universidad del Norte de Colombia.

Meliá J, Peiró J (2016). La medida de la satisfacción laboral en contextos organizacionales de salud. Valencia-España: Universidad de Valencia. 
Milagros Lissette Millones Trinidad

Mesa L, Romero M (2016). Profesionales de enfermería y cuidado en las condiciones laborales actuales (Tesis). Bogotá-Colombia: Pontificia Universidad Javeriana.

Ministerio de Salud del Perú (2018). Norma técnica sobre las condiciones de trabajo en el ámbito hospitalario por las(os) enfermeras(os). Lima-Perú: MINSA.

Ministerio de Trabajo y Promoción del Empleo del Perú (2018). Condiciones laborales y satisfacción laboral en el personal de enfermería. Lima-Perú: MTPE.

Ñiquen M (2017). Satisfacción Laboral en los Trabajadores de la Institución Estatal Gerencia Regional de Salud - La Libertad. Lima-Perú: MINSA.

Organización Internacional del Trabajo (2018). Condiciones de trabajo en los trabajadores de enfermería o salud. Ginebra-Suiza: OIT.

Organización Internacional del Trabajo (2018). Condiciones de trabajo físicas hospitalarias del personal de enfermería. Ginebra-Suiza: OIT; 2018.

Organización Internacional del Trabajo (2018). Condiciones de trabajo psicológicas hospitalarias del personal de enfermería. Ginebra-Suiza: OIT.

Organización Internacional del Trabajo (2018). Condiciones de trabajo sociales hospitalarias del personal de enfermería. Ginebra-Suiza: OIT.

Organización Internacional del Trabajo (2018). Reporte estadístico de condiciones de trabajo a nivel mundial de los trabajadores de salud. Ginebra-Suiza: OIT.

Organización Mundial de la Salud, Consejo Internacional de Enfermería y Organización Internacional del Trabajo (2018). Análisis de las condiciones de trabajo en los profesionales de enfermería. Ginebra-Suiza: OMS/CIE/OIT.

Quintana M, Valenzuela S, Paravic T (2016). Enfermería desde la perspectiva del trabajo decente (Tesis). Santiago-Chile: Universidad de Concepción de Chile.

Reyes A (2018). Relación entre las condiciones laborales y la satisfacción laboral de los colaboradores de la Comisión Nacional para el Desarrollo y Vida sin Drogas Oficina Zonal Tarapoto. Tarapoto-Perú.

Tomasina F, Bozzo E, Chaves E, Pucci F (2018). Impacto de las condiciones laborales en la salud de trabajadores de un centro quirúrgico (Tesis). Montevideo-Uruguay: Universidad de la República de Uruguay. 